\title{
Square Planar 3 Molecular Geometry
}

National Cancer Institute

\section{Source}

National Cancer Institute. Square Planar 3 Molecular Geometry. NCI Thesaurus. Code C103213.

Square planar molecular geometry where the Cahn-Ingold-Prelog (CIP) priority of the donor atom directly across from the priority 1 donor atom is 3 . 